\title{
Intraoperative awareness during laparoscopic sleeve gastrectomy
}

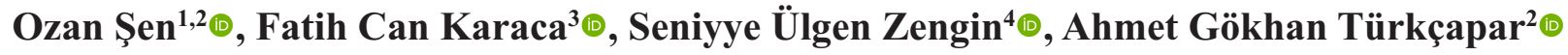 \\ ${ }^{1}$ Department of Health Sciences, Nişantaşı University, İstanbul, Turkey \\ ${ }^{2}$ Türkçapar Bariatrics, Center for Obesity Surgery, Istanbul, Turkey \\ ${ }^{3}$ Department of Health Sciences, Bilgi University, İstanbul, Turkey \\ ${ }^{4}$ Department of Anesthesiology and Reanimation, Bezmialem Vakif University, Faculty of Medicine, Istanbul, Turkey
}

\begin{abstract}
Objectives: The aim of this study is to determine the incidence of intraoperative awereness (IA) in our patients who underwent laparoscopic sleeve gastrectomy (LSG) and the factors affecting the formation of this complication.
\end{abstract}

Methods: Four hundred ten patients who underwent LSG between March 2018 and September 2020 were included in the study. By April 2019, we started using the Bispectral index (BIS) monitoring, which measures the depth of anesthesia in all of our LSG cases $(n=167)$. Patients with and without BIS monitorization were divided into two groups and compared.

Results: In our series, IA was seen in 3 patients ( 2 males) in two different hospitals $(0.7 \% ; n=410)$. They were all in the non BIS group $(n=243)$. The median duration of anesthesia was 120 minutes (ranging 90 180 ) in the non-BIS, and 113 minutes (ranging, 90-140) in the BIS group $(p<0.001)$. Hypotension developed in 63 patients in non-BIS and 12 patients in BIS group at the beginning of the operation $(<90 / 60 \mathrm{~mm} \mathrm{Hg})$. The total remifentanil infusion dose administered during the anesthesia period in the BIS group was $1310 \pm$ $351 \mathrm{mcg}$, and $1330 \pm 270 \mathrm{mcg}$ in the non BIS group $(p=0.002)$. The effect of BIS monitorization between groups on IA, did not show statistical significance $(p=0.27)$.

Conclusions: Anesthesia techniques that work well for patients with normal weight may not be safe and appropriate for obese patients. Especially in patients with intraoperative hypotension, it is necessary to be more careful about dose adjustment of anesthetic drugs.

Keywords: Bariatric surgery, sleeve gastrectomy, general anesthesia, complication, intraoperative awareness

B ariatric surgery is one of the most effective treatment for morbid obesity. As obesity increases worldwide, people turning to bariatric surgery for weight loss also increases [1]. With increase in bariatric surgery, anesthesiologists face a new challenge in terms of management of obese patients during surgery.
Since the dose adjustment of the intravenous anesthetic agents specified in the anesthesia guidelines is usually based on the total body weight of people with normal weight, anesthesia management of morbidly obese patients requires extra care. Although there are no definitive guidelines for the dose adjustment of intravenous anesthetic agents in morbidly obese patients 
$[2,3]$, it is generally recommended to adjust the dose according to lean body mass $[4,5]$. Many comorbidities such as diabetes, hypertension, left ventricular hypertrophy, sleep apnea, and pulmonary hypertension accompany morbid obese people undergoing bariatric surgery. These comorbidities together with the excess adipose tissue and the lipophilic properties of many drugs used in general anesthesia affect the pharmacokinetics and pharmacodynamics of anesthetics, causing the drugs to show their effects in a very narrow spectrum [6]. In this respect, anesthesia management of bariatric surgery patients gains the utmost importance.

The patient regaining consciousness during general anesthesia is defined as awareness. Awareness is a serious condition that prompts patients into a panic situation even if they do not feel any pain at that time [7]. These patients experience insomnia, recurrent nightmares and post-traumatic stress disorder in postliminary periods $[8,9]$. Intraoperative awareness (IA) is a rare but serious complication rating between $0.1-0.2 \%$ [10]. The most common cause is superficial anesthesia and the most common disciplines IA observed are in obstetrics and cardiac anesthesia. The ratio of IA was reported to be $1 \%$ in the high-risk cases $[11,12]$. Obesity is defined to be an independent risk factor for IA $[5,13]$.

The aim of this study was to determine the incidence of IA in our patients undergoing laparoscopic sleeve gastrectomy (LSG), and the factors contributing to the development of this complication. To our knowledge, this is the first study in the literature reporting that IA might be observed during LSG.

\section{METHODS}

Four hundred ten patients ( $56 \%$ female) who underwent primary LSG between March 2018 and September 2020 were included in the study.

\section{Preoperative Work-up}

Each patient underwent a thorough evaluation of laboratory tests, upper GI endoscopy, and abdominal ultrasonography before the surgery. Also, patients were evaluated by a cardiologist, a pulmonology, and an anesthesiologist as a part of routine work-up.
Anesthesia Management and Surgical Technique

LSG was applied to all patients using the same surgical technique. The operations were performed in two different hospitals with two different anesthesiology teams, using the same anesthesia protocol. Table 1 summarizes the doses and durations of the anesthetic agents administered during the procedures. Following the tracheal intubation and inhalation anesthesia, a 12 mm optical trocar (Endopeth $\mathrm{Xcel}^{\circledR}$ ) was inserted into the abdomen from the left subcostal area under direct vision and abdomen was insufflated. LSG was performed using five trocar technique after applying a 45 degrees reverse Trendelenburg (modified litotomy) position. After LSG was completed, the staple line was oversewn using 3.0 V-Loc ${ }^{\mathrm{TM}}$ suture in all patients.

\section{Evaluation of Intraoperative Awareness}

In order to evaluate IA after surgery, each patient was questioned in terms of whether they remembered anything, heard anything or felt any pain during the operation.

\section{Bispectral Index Monitoring}

The use of Bispectral Index monitoring equipment (BIS ${ }^{\mathrm{TM}}$ Medtronic), which monitors the depth of anesthesia was initiated for all of our bariatric surgery cases $(n=167)$ starting in April 2019. BIS moniterization was not used in any of our LSG cases before April 2019.

Patients with and without BIS monitoring were divided into two groups (Non-BIS and BIS group). BIS values between 40 and 60 indicate adequate anesthesia depth for the surgery.

Patients who developed intraoperative hypotension (systolic blood pressure below $90 \mathrm{mmhg}$ ) in both groups were recorded. Two groups compared in terms of demographics, the rate of IA, the factors affecting the condition, and the effect of BIS monitorization.

\section{Exclusion Criteria}

Cases that included additional surgical procedures such as cholecystectomy and other bariatric procedures were excluded from this study in order to avoid skewness in the operation and anesthesia time. Patients with a previous history of IA were excluded from the study.

The study was approved by the institutional ethics commitee (ATADEK- 2019-17/11). All patients were 
informed about the study in details, and written informed consents were obtained. All data, including intraoperative vital parameters were recorded prospectively and analyzed retsospectively.

\section{Statistical Analysis}

Statistical analysis was performed using SPSS (version 21, SPSS, Inc., Chicago, IL, USA). Standard deviation and mean values were used for the variables with normal distribution and median values were used for the variables that were not normally distributed. Chi-square or Fisher's exact tests were used for categorical variables; while for continuous variables, independent-samples T-test or Mann-Whitney U test were performed. $P$ values $<0.05$ were considered statistically significant.

\section{RESULTS}

Between March 2018 and September 2020 four hundred ten patients ( $56 \%$ female) with a mean age of $37.4 \pm 11.6$ years, a median body mass index (BMI) of $41.3 \mathrm{~kg} / \mathrm{m}^{2}$ (33-67) and ASA score of 2-3 had undergone LSG. In our series, IA was observed in three patients ( 2 male, 1 female) in two different hospitals $(0.7 \%)$. Three of them were in the non BIS group $(\mathrm{n}=$ 243). The median duration of anesthesia was 120 minutes (90-180) in the non BIS group and 113 minutes (90-140) in the BIS group $(p<0.001)$.

Hypotension developed in 63 patients in non-BIS, and 12 patients in BIS group at the beginning of the operation $(<90 / 60 \mathrm{~mm} \mathrm{hg})$. The total remifentanil infusion dose administered during the anesthesia period in the BIS group was $1310 \pm 351 \mathrm{mcg}$, and $1330 \pm 270$ mcg in the non BIS group $(p=0.002)$. The demographic characteristics of the groups are shown in Table 2.

The first patient who experienced IA was 41 years old female with a BMI of $39 \mathrm{~kg} / \mathrm{m}^{2}$. The patient stated that she woke up at the beginning of the surgery, was fully awake, heard everything spoken during sterilization and surgical draping, wanted to say that she was awake. She was very scared of the situation and she was not able to say anything or move. She added that this situation lasted for 3-4 minutes, and she could not remember what happened subsequently.

The other two patients were males aged 40 years with a BMI of $46 \mathrm{~kg} / \mathrm{m}^{2}$ and $44 \mathrm{~kg} / \mathrm{m}^{2}$ ). Their experiences were very similar. According to their statements, awakenings were somewhere during the beginning of the surgery. Both patients experienced similar feelings of great pressure in the abdomen, surgical manipulation such as trocar entrance, which they described specifically as "there was a drill going into my abdomen". They emphasized that they felt enormous pain and this "nightmare" lasted for 10-15 minutes.

Except for a temporary period of hypotension at the beginning of the surgery, there was no abnormality in vital signs suggestive of IA in all 3 patients. Two male patients received psychotherapy support for 6 months due to post-traumatic stress disorder.

\section{Table 1. Applied anesthesia regimen for LSG patients}

\begin{tabular}{ll}
\hline Local anesthetic (Lidocaine) & $1 \mathrm{mg} / \mathrm{kg}$ \\
Propofol & $1.5-2 \mathrm{mg} / \mathrm{kg}$ bolus \\
Fentanyl & $1-1.5 \mu \mathrm{g} / \mathrm{kg}$ bolus \\
Rocuronium bromid & $\begin{array}{l}0.6-0.8 \mathrm{mg} / \mathrm{kg} \text { bolus } \\
\text { *repeated dosage intraoperativley every 20-30 minutes }\end{array}$ \\
Trakeal entubation & $2 \%$ \\
\hline Sevoflurane & $10-25 \mathrm{ml} / \mathrm{hour}$ infusion \\
Remifentanil (50 mcg/ml) & \\
First trocar insertion and CO2 insuflation & \\
Reverse trendelenburg position & \\
Begin to operation & \\
\hline LSG = Laparoscopic sleeve gastrectomy &
\end{tabular}


Table 2. Patients' characteristics

\begin{tabular}{lccc}
\hline Sleeve gastrectomy & $\begin{array}{c}\text { Without BíS } \\
(\mathbf{n}=\mathbf{2 4 3})\end{array}$ & $\begin{array}{c}\text { With BíS } \\
(\mathbf{n}=\mathbf{1 6 7})\end{array}$ & p value \\
\hline Male/female & $118 / 125$ & $62 / 105$ & 0.02 \\
\hline Age (years) & $37.5 \pm 11.5$ & $37.2 \pm 11.8$ & 0.78 \\
\hline Preoperative weight $(\mathrm{kg})$ & $121(88-215)$ & $113(87-215)$ & 0.001 \\
\hline $\begin{array}{l}\text { Preoperative body mass index } \\
\left(\mathrm{kg} / \mathrm{m}^{2}\right)\end{array}$ & $42.4(33-67)$ & $39.7(32-62)$ & 0.001 \\
\hline ASA score 2 & 81 & 88 & 0.001 \\
\hline ASA score 3 & 162 & 79 & 0.001 \\
\hline Anesthesia time (minutes) & $120(90-180)$ & $113(90-140)$ & 0.001 \\
\hline Intraoperative hypotension $(<90$ & 63 & 12 & \\
mm Hg) & $1330 \pm 270$ & $1310 \pm 351$ & 0.002 \\
\hline Total remifentanil dosage $(\mathrm{mcg})$ & $3(1.2 \%)$ & 0 & 0.27 \\
\hline Awereness & & & 0.9 \\
\hline Comorbidities $(+)$ & 32 & 21 & 0.76 \\
\hline \multicolumn{1}{c}{ Type 2 diabetes } & 194 & 127 & 0.18 \\
\hline İnsüline resistance & 68 & 37 & 0.06 \\
\hline Hypertension & 132 & 75 & 0.63 \\
\hline Dyslipidemia & 59 & 44 & \\
\hline Sleep apnea & & & \\
\hline
\end{tabular}

$\mathrm{BIS}=$ Bispectral index

\section{DISCUSSION}

IA is a rare but serious complication with an incidence of $1 \%$ for high-risk individuals $[11,13]$. In our study, IA was developed in three $(0.7 \%)$ patients and there were all at the beginning of the operation. One patient with ASA $2(n=169)$ and two patients with ASA $3(n=241)$ experienced IA. We did not find a significant effect of the ASA score on IA $(p=0.20)$.

There are no definitive guidelines for dose adjustment of intravenous anesthetic agents in obese patients. Propofol is a highly lipophilic substance commonly used in anesthesia induction. While dose adjustment is performed according to the total body weight in normal-weight people, this approach should be avoided as it can easily cause adverse effects in obese people.

Some studies indicate that the current dose of propofol administered during induction in obese individuals is insufficient [14-16]. Agents used in the induction of anesthesia redistributes quicker in the fat tissue. The meaning of this is that, obese patients awake earlier than non-obese people with single bolus application.

Anesthetic drugs such as propofol, remifentanil have a great hypotensive potential in higher doses. The reverse Trendelenburg or modified litotomy position for LSG induces the hypotensive effect of anesthetic drugs. İntraoperative hypotension is mostly undesirable by the surgeons. In most cases, it is preferable that the patient is normotensive in order to observe any signs of bleeding from the staple line or other surgical sites. It is worth mentioning that the caution of the anesthesiology team regarding the hypotension that might occur as a result of the surgical position at the beginning of the operation is one of the most important points to be considered for the prevention of this complication. Anesthesiologists' adjustment process of dose regulation in an attempt to avoid subsequent hypotension might be a factor in the manifestation of IA. In our study we also experienced intraoperative hypotension in our patients who underwent LSG. Hy- 
potension developed in 63 patients in non-BIS, and 12 patients in BIS group at the beginning of the operation $(<90 / 60 \mathrm{~mm} \mathrm{Hg})$.

Nowadays, electroencephalogram-like devices that monitor brain functions are frequently used during surgery. The most commonly used is the BIS monitorisation, which measures the patient's level of consciousness by processing an electroencephalographic signal obtained from a single frontal electrode. The BIS interval is determined between 100-0, defining the complete awakeness and the absence of brain activity. BIS values between 40 and 60 indicate adequate anesthesia depth for the surgery whereas values below 40 indicate the deep hypnotic phase. It is recommended to keep the target value in the interval of 40-60 to prevent IA [17]. In our study, patients in the BIS group, median BIS score was 36 (26-47) during the operation.

The data on the effect of BIS monitorisation on the prevention of IA is controversial. The B-aware study reported that BIS monitorization provides a $0.74 \%$ of risk reduction in IA in high-risk individuals [18]. In a large multicentric cohort study, it was reported that BIS monitorization did not provide a positive effect on the IA [5].

In our LSG series, we experienced a fairly high rate of IA in three $(0.7 \%)$ of our patients. All of these cases were in the non BIS group $(n=243 ; 1.2 \%)$. No incident of IA was observed in the BIS group. While we evaluated our patients for the effect of BIS monitorization on IA, the ratio did not show statistical significance $(p=0.27)$.

\section{CONCLUSION}

Many factors affect the pharmacokinetics and pharmacodynamics of the anesthetics in obese patients. Anesthesiology practices that work well for patients of normal weight may not be safe and adequate for obese patients. Especially in patients with intraoperative hypotension, it is necessary to be more careful about dose adjustment of anesthetic drugs. Comprehensive studies are needed to create definitive guidlines in this area.

\section{Authors' Contribution}

Study Conception: OŞ, AGT; Study Design: OŞ, FCK, AGT; Supervision:; Funding: OŞ, AGT; Mate- rials: ED, SK; Data Collection and/or Processing: OŞ, AGT; Statistical Analysis and/or Data Interpretation: OŞ; Literature Review: OŞ, FCK, SÜZ, AGT; Manuscript Preparation: OŞ, AGT and Critical Review: OŞ, FCK, SÜZ, AGT.

\section{Conflict of interest}

The authors disclosed no conflict of interest during the preparation or publication of this manuscript.

\section{Financing}

The authors disclosed that they did not receive any grant during the conduction or writing of this study.

\section{REFERENCES}

1. English WJ, DeMaria EJ, Brethauer SA, Mattar SG, Rosenthal RJ, Morton JM. American Society for Metabolic and Bariatric Surgery estimation of metabolic and bariatric procedures performed in the United States in 2016. Surg Obes Relat Dis 2018; $14: 259-63$.

2. Green B, Duffull SB. What is the best size descriptor to use for pharmacokinetic studies in the obese? Br J Clin Pharmacol 2004;58:119-33.

3. Morgan DJ, Bray KM. Lean body mass as a predictor of drug dosage. Implications for drug therapy. Clin Pharmacokinet 1994;26:292-307.

4. Ingrande J, Brodsky JB, Lemmens HJ. Lean body weight scalar for the anesthetic induction dose of propofol in morbidly obese subjects. Anesth Analg 2011;113:57-62.

5. Ingrande J, Lemmens HJ. Dose adjustment of anaesthetics in the morbidly obese. Br J Anaesth 2010;105(Suppl 1):i16-23.

6. Nightingale CE, Margarson MP, Shearer E, Redman JW, Lucas DN, Cousins JM, et al. Peri-operative management of the obese surgical patient 2015: Association of Anaesthetists of Great Britain and Ireland Society for Obesity and Bariatric Anaesthesia. Anaesthesia 2015;70:859-76.

7. Moerman N, Bonke B, Oosting J. Awareness and recall during general anesthesia. Anesthesiology 1993;79:454-64.

8. Osterman JE, Van der Kolk A. Awareness during anesthesia and posttraumatic stress disorder. Gen Hosp Psychiatry 1998;50:274-8.

9. Domino KB, Posner KL, Caplan RA, Cheney FW. Awareness during anesthesia: a closed claims analysis. Anesthesiology 1999;90:1053-61.

10. Sebel P, Bowdle T, Ghoneim M, Rampil I, Padilla R, Gan T, et al. The incidence of awareness during anesthesia: a multicenter United States study. Anesth Analg 2004;99:833-9.

11. Paech MJ, Scott KL, Clavisi O, Chua S, McDonnell N, The ANZA Trials Group. A prospective study of awareness and recall associated with general anaesthesia for Caesarean section. Int $\mathbf{J}$ Obstet Anesth 2008;17:298-303.

12. Ranta S, Jussila J, Hynynen M. Recall of awareness during 
cardiac anaesthesia: influence of feedback information to the anaesthesiologist. Acta Anaesthesiol Scand 1996;40:554-60. 13. Ghoneim MM, Block RI, Haffarnan M, Mathews MJ. Awareness during anesthesia: risk factors, causes and sequelae: a review of reported cases in the literature. Anesth Analg 2009;108:52735.

14. Pandit JJ, Andrade J, Bogod DG, Hitchman JM, Jonker WR, Lucas N, et al.; Royal College of Anaesthetists; Association of Anaesthetists of Great Britain and Ireland. The 5th National Audit Project (NAP5) on accidental awareness during general anaesthesia: protocol, methods and analysis of data. Anaesthesia 2014;69:1078-88.

15. Subramani Y, Riad W, Chung F, Wong J. Optimal propofol induction dose in morbidly obese patients: A randomized con- trolled trial comparing the bispectral index and lean body weight scalar. Can J Anesth 2017;64:471-9.

16. Cao Y-H, Chi P, Zhao Y-X, Dong X-C. Effect of bispectral index-guided anesthesia on consumption of anesthetic and eraly postoperative cognitive dysfunction after liver transplantation. Medicine 2017:96:e7966.

17. Punjasawadwong Y, Boonjeungmonkol N, Phongchiewboon A. Bispectral index for improving anaesthetic delivery and postoperative recovery. Cochrane Database Syst Rev 2007;4:CD003843- CD003843

18. Myles PS, Leslie K, McNeil J, Forbes A, Chan MT. Bispectral index monitoring to prevent awareness during anaesthesia: the B-Aware randomised controlled trial. Lancet 2004;363:1757-63 